\section{Connective Stability for Large Scale Systems Described by Functional Differential Equations}

RUAN SHIGUI

Abstract-In this note we employ the decomposition-aggregation method, the comparison principle, and vector Lyapunov functionals to investigate the stability of large scale systems described by functional differential equations under structural perturbations. Some sufficient conditions are given such that the zero solution is connectively and uniformly asymptotically stable. An example to illustrate the main results is given.

\section{INTRODUCTION}

During the last decade, quite a few authors have been concerned with the qualitative analysis of large scale systems, especially stability analysis. Many of the results dealing with stability can be found in the books by Michel and Miller [7] and Siljak [9].

The connective stability concept was introduced in [2], [8] to study large scale systems, and was developed by Ladde [4] for functional differential systems. In this note the decomposition-aggregation method, the comparison principle, and vector Lyapunov functionals are used to derive sufficient conditions for stability of large scale systems described by functional differential equations under structural perturbations. Some results of Michel and Miller [7] are extended to the connectiveness property of stability.

\section{NOTATION}

Let $R^{n}$ denote Euclidean $n$-space, let $x^{T}=\left(x_{1}, x_{2}, \cdots, x_{n}\right)$ denote the transpose of $x \in R^{n}$, let $J=\left[t_{0},+\infty\right), t_{0} \geq 0$.

A wedge is a continuous function $W:[0, \infty) \rightarrow[0, \infty)$ such that $W(0)$

$=0, W(r)$ is strictly increasing and $W(r) \rightarrow \infty$ as $r \rightarrow \infty$.

For given $r>0, H>0$, let $C_{n}=C\left([-r, 0], R^{n}\right)$, for $\phi \in C_{n}$, define $\|\phi\|=\sup _{-r \leqslant \theta \leqslant 0}|\phi(\theta)|$, let $C_{n}^{H}=\left\{\phi \in C_{n}:\|\phi\|<H\right\}$.

Consider the large scale systems described by functional differential equations

$$
\dot{x}(t)=f\left(t, x_{t}\right)
$$

where $f: J \times C_{n}^{H} \rightarrow R^{n}$ is continuous and $f(t, 0)=0$. We suppose that for every $\phi \in C_{n}$ and for every $t_{0} \geq 0$, system (1) possesses a unique solution $x_{t}\left(t_{0}, \phi\right)$ with $x_{t_{0}}=\phi$ and we denote by $x(t)=x\left(t ; t_{0}, \phi\right)$ the value of $x_{t}\left(t_{0}, \phi\right)$ at $t$.

Let $V(t, \phi)$ be a continuous nonnegative functional defined on $J \times C_{n}^{H}$, the upper right-hand derivative of $V$ along the solution of (1) is defined as

$$
D_{(1)} V(t, \phi)=\lim _{h \rightarrow 0^{+}} \sup 1 / h\left\{V\left(t+h, x_{t+h}\left(t_{0}, \phi\right)\right)-V\left(t, x_{t}\left(t_{0}, \phi\right)\right)\right\} .
$$

Now, we decompose (1) into $m$ interconnection subsystems described by the equations

$$
\dot{x}^{i}(t)=h_{i}\left(t, x_{i}^{i}\right)+g_{i}\left(t, x_{t}\right)
$$

where $i \in I_{m} \stackrel{\text { def }}{=}\{1,2, \cdots, m\}, \Sigma_{i=1}^{m} n_{i}=n, f_{i} \in C\left(J \times C_{n_{i}}^{H_{i}}, R^{n_{i}}\right), g_{i}$ $\in C\left(J \times C_{n}^{H}, R^{n_{i}}\right)$.

We assume that interconnection functions $g_{i}\left(t, x_{t}\right)$ depend on the $m \times$ $m$ interconnection matrices $E_{t}=\left(e_{i}^{i j}\right)$

$$
g_{i}\left(t, x_{t}\right)=g_{i}\left(t, e_{t}^{i 1} x_{t}^{1}, e_{t}^{i 2} x_{t}^{2}, \cdots, e_{t}^{i m} x_{t}^{m}\right)
$$

for $i \in I_{m}$, where $e_{,}^{i j} \in C([-r, 0],[0,1])$ are coupling functions which

Manuscript received April 8, 1987; revised June 23, 1987.

The author is with the Department of Mathematics, Huazhong Normal University, Wuhan, China.

IEEE Log Number 8716719 are elements of an $m \times m$ hereditary interconnection matrix function $E_{t}$, $e^{i j} x^{i}=e^{i j}(t+\theta) x^{i}(t+\theta)$ for $\theta \in[-r, 0]$

When $E_{t}=0$, from (3) we obtain the isolated subsystem

$$
\dot{x}^{i}(t)=h_{i}\left(t, x_{t}^{i}\right) \text {. }
$$

\section{CONNECTIVE STABILITY}

Theorem 1: Suppose that the following conditions are satisfied.

i) There exist functional $V(t, \phi) \in C\left(J \times C_{n}^{H}, R_{+}^{m}\right), V(t, 0)=0$, $V(t, \phi)$ is locally Lipschitzian in $\phi$ for $t \in J$; two wedges $W_{1}, W_{2}$, and positive constants $\alpha_{i}$ such that

$$
W_{1}(\|\phi\|) \leqslant \sum_{i=1}^{m} \alpha_{i} V_{i}(t, \phi) \leqslant W_{2}(\|\phi\|)
$$

for all $t \in J, \phi \in C_{n}^{H}$, where

$$
V(t, \phi)=\left(V_{1}(t, \phi), V_{2}(t, \phi), \cdots, V_{m}(t, \phi)\right)^{T} .
$$

ii) There exist a function $w \in C\left(J \times R_{+}^{m}, R_{+}^{m}\right), w(t, 0)=0$, and $w(t$, $u$ ) is quasi-monotone nondecreasing in $u$, and

$$
D_{(1)} V(t, \phi) \leqslant w(t, V(t, \phi))
$$

for all $(t, \phi) \in J \times C_{n}^{H}$ and all interconnection matrices $E_{t} \in \bar{E}_{t}$.

iii) The zero solution $u=0$ of the comparison system

$$
\dot{u}(t)=w(t, u), u\left(t_{0}\right)=u_{0}>0
$$

is uniformly asymptotically stable.

Then the zero solution of system (1) is connectively and uniformly asymptotically stable.

The proof of Theorem 1 is similar to the proof of Theorem 3 of [4]; here we omit it.

Now, we consider the system (1) with decompositions (2) and (3).

Definition: The isolated subsystem (4) possesses property $A$, if:

i) there exist a functional $V_{i}\left(t, \phi^{i}\right) \in C\left(J \times C_{n_{i}}^{H_{i}}, R_{+}^{\left.m_{i}\right)}\left(1 \leqslant m_{i} \leqslant\right.\right.$ $\left.n_{i}\right), V_{i}(t, 0)=0, V_{i}\left(t, \phi^{i}\right)$ is locally Lipschitzian in $\phi^{i}$, and two wedges $W_{i 1}, W_{i 2}$ such that

$$
W_{i 1}\left(\left\|\phi^{i}\right\|\right) \leqslant\left\|V_{i}\left(t, \phi^{i}\right)\right\| \leqslant W_{i 2}\left(\left\|\phi^{i}\right\|\right)
$$

for all $\left(t, \phi^{i}\right) \in J \times C_{n_{i}}^{H_{i}}, i \in I_{m}$, where

$$
V_{i}\left(t, \phi^{i}\right)=\left(V_{i 1}\left(t, \phi^{i}\right), V_{i 2}\left(t, \phi^{i}\right), \cdots, V_{i m_{i}}\left(t, \phi^{i}\right)\right)^{T} ;
$$

ii) there exist constants $\sigma_{i} \in R_{+}$, a wedge $W_{i 3}$, and a function $w_{i s} \in$ $C\left(J \times R_{+}^{m}, R_{+}\right), w_{i s}(t, 0)=0$, and $\omega_{i s}(t, u)$ is quasi-monotone nondecreasing in $u$ such that

$$
D_{(4)} V_{i}\left(t, \phi^{i}\right) \leqslant w_{i s}\left(t, \bar{V}_{i}\left(t, \phi^{i}\right)\right)+\sigma_{i} W_{i 3}\left(\left\|\phi^{i}\right\|\right)
$$

where $m_{0}=\max _{i \in I_{m}}\left\{m_{i}\right\}, \bar{V}_{i}=\left(V_{i 1}, V_{i 2}, \cdots, V_{i m_{0}}\right)^{T}$

$$
V_{i s}=\left\{\begin{array}{cc}
V_{i s} & s \leqslant m_{i} \\
0 & s>m_{i}
\end{array} i \in I_{m}, s \in I_{m_{0}} .\right.
$$

If, in addition, $V_{i}\left(t, \phi^{i}\right)$ is continuously differentiable on $J \times C_{n_{i}}^{H_{i}}$, then the isolated subsystem (4) possesses property $A_{1}$.

Theorem 2: If the following conditions are satisfied:

i) each isolated subsystem (4) possesses property $A$;

ii) there exist constants $a_{i j} \in R_{+}$such that

$g_{i}\left(t, e_{t}^{i 1} x_{t}^{1}, e_{t}^{i 2} x_{t}^{2}, \cdots, e_{t}^{i m} x_{t}^{m}\right) \leqslant\left(W_{i 3}\left(\left\|\phi^{i}\right\|\right)\right)^{1 / 2}$

$$
\sum_{i=1}^{m}\left\|\bar{e}_{i}^{i j}\right\| a_{i j}\left(W_{j 3}\left(\left\|\phi^{j}\right\|\right)\right)^{1 / 2}
$$

for all $\left(t, \phi^{i}\right) \in J \times C_{n_{i}}^{H_{i}}, i \in I_{m}$ and all interconnection matrices $E_{t} \in$ $\bar{E}_{i}$;

0018-9286/88/0200-0198\$01.00 C 1988 IEEE 
iii) the $m \times m$ text matrix $S=\left(s_{i j}\right)$ defined by

$$
s_{i j}= \begin{cases}-\sigma_{i}-\left\|\bar{e}_{t}^{i n}\right\| L_{i} a_{i i} & i=j \\ -1 / 2\left(\left\|\bar{e}_{t}^{i j}\right\| L_{i} a_{i j}+\left\|\bar{e}_{t}^{j i}\right\| L_{j} a_{j i}\right) & i \neq j\end{cases}
$$

has positive successive principal minors, where $L_{i}$ is the Lipschitz constant of $V_{i}\left(t, \phi^{i}\right)$ in $\phi^{i}$;

iv) the zero solution $u=0$ of the comparison system

$$
\dot{u}(t)=\bar{w}(t, u), u\left(t_{0}\right)=u_{0}>0
$$

is uniformly asymptotically stable, where

$$
\begin{gathered}
\bar{w}(t, u)=\left(\sum_{i=1}^{m} w_{i 1}(t, u), \sum_{i=1}^{m} w_{i 2}(t, u), \cdots, \sum_{i=1}^{m} w_{i m_{0}}(t, u)\right)^{T}, \\
w_{i s}(t, u)=\left\{\begin{array}{cc}
w_{i s} & s \leqslant m_{i} \\
0 & s>m_{i}
\end{array} \quad i \in I_{m}, s \in I_{m_{0}} .\right.
\end{gathered}
$$

Then the zero solution of large scale system (1) with decomposition (2) and (3) is connectively and uniformly asymptotically stable.

Proof: We choose a Lyapunov functional

$$
\begin{aligned}
V(t, \phi) & =\sum_{i=1}^{m} \bar{V}_{i}\left(t, \phi^{i}\right) \\
& =\left(\sum_{i=1}^{m} V_{i 1}\left(t, \phi^{i}\right), \sum_{i=1}^{m} V_{i 2}\left(t, \phi^{i}\right), \cdots, \sum_{i=1}^{m} V_{i m_{0}}\left(t, \phi^{i}\right)\right)^{T}
\end{aligned}
$$

obviously, $V(t, \phi)>0$ and

$$
\begin{aligned}
\|V(t, \phi)\| & =\sum_{s=1}^{m_{0}}\left\{\sum_{i=1}^{m} V_{i s}\left(t, \phi^{i}\right)\right\} \\
& =\sum_{i=1}^{m}\left\{\sum_{s=1}^{m_{i}} V_{i s}\left(t, \phi^{i}\right)\right\}=\sum_{i=1}^{m}\left\|V_{i}\left(t, \phi^{i}\right)\right\| .
\end{aligned}
$$

Since each isolated subsystem (4) possesses property $A$, it follows that

$$
\sum_{i=1}^{m} W_{i 1}\left(\left\|\phi^{i}\right\|\right) \leqslant\|V(t, \phi)\| \leqslant \sum_{i=1}^{m} W_{i 2}\left(\left\|\phi^{i}\right\|\right)
$$

then there exist wedges $W_{1}, W_{2}$ such that

$$
W_{1}(\|\phi\|) \leqslant\|V(t, \phi)\| \leqslant W_{2}(\|\phi\|) .
$$

Consider

$$
\begin{aligned}
D_{(2)} V(t, \phi) & =\sum_{i=1}^{m} D_{(2)} \bar{V}_{i}\left(t, \phi^{i}\right) \\
& =\left(D_{(2)} V_{\mathrm{l}}(t, \phi), D_{(2)} V_{2}(t, \phi), \cdots, D_{(2)} V_{m_{0}}(t, \phi)\right)^{T} ;
\end{aligned}
$$

we have

$$
\begin{aligned}
D_{(2)} V_{s}(t, \phi) & =\sum_{i=1}^{m} D_{(2)} V_{i s}\left(t, \phi^{i}\right) \\
& \leqslant \sum_{i=1}^{m}\left\{D_{(4)} V_{i s}\left(t, \phi^{i}\right)+L_{i}\left\|g_{i}\left(t, e_{t}^{i !} \phi^{1}, e_{t}^{i 2} \phi^{2}, \cdots, e_{t}^{i m} \phi^{m}\right)\right\|\right\}
\end{aligned}
$$

since

$$
\begin{aligned}
V & =\left(V_{1}, V_{2}, \cdots, V_{m_{0}}\right)^{T}=\left(\sum_{i=1}^{m} V_{i 1}, \sum_{i=1}^{m} V_{i 2}, \cdots, \sum_{i=1}^{m} V_{i m_{0}}\right)^{T} \\
& \geqslant\left(V_{i 1}, V_{i 2}, \cdots, V_{i m_{0}}\right)^{T}=\bar{V}_{i}
\end{aligned}
$$

by hypotheses i) and ii), we have

$$
\begin{aligned}
D_{(2)} V(t, \phi) \leqslant & \sum_{i=1}^{m} w_{i s}\left(t, \bar{V}_{i}\right)+\sum_{i=1}^{m} \sigma_{i} W_{i 3}\left(\left\|\phi^{i}\right\|\right) \\
& +\sum_{i=1}^{m} L_{i}\left(W_{i 3}\left(\left\|\phi^{i}\right\|\right)\right)^{1 / 2} \sum_{j=1}^{m}\left\|\bar{e}_{i}^{i j}\right\| a_{i j}\left(W_{j 3}\left(\left\|\phi^{j}\right\|\right)\right)^{1 / 2} \\
\leqslant & w_{i s}(t, V)-P
\end{aligned}
$$

where

$P=\sum_{i=1}^{m}\left\{-\sigma_{i} W_{i 3}\left(\left\|\phi^{i}\right\|\right)-\left(W_{i 3}\left(\left\|\phi^{i}\right\|\right)\right)^{1 / 2} \sum_{j=1}^{m} L_{j}\left\|\bar{e}_{t}^{i j}\right\| a_{i j}\left(W_{j 3}\left(\left\|\phi^{j}\right\|\right)\right)^{1 / 2}\right\}$.

Let

$$
\begin{gathered}
W=\left(W_{13}^{1 / 2}\left(\left\|\phi^{\dagger}\right\|\right), W_{23}^{1 / 2}\left(\left\|\phi^{2}\right\|\right), \cdots, W_{m 3}^{1 / 2}\left(\left\|\phi^{m}\right\|\right)\right)^{T}, R=\left(r_{i j}\right)_{m \times m} \\
r_{i j}=\left\{\begin{array}{cc}
-\sigma_{i}-L_{i}\left\|\bar{e}_{t}^{i i}\right\| a_{i i} & i=j \\
-L_{i}\left\|\bar{e}_{i}^{i j}\right\| a_{i j} & i \neq j
\end{array}\right.
\end{gathered}
$$

Thus

$$
P=W^{T}\left(\left(R+R^{T}\right) / 2\right) W \stackrel{\text { def }}{=} W^{T} S W
$$

where $S$ is the text matrix given in hypothesis iii); by hypothesis iii) we know that $P$ is semipositive definite, then

$$
D_{(2)} V_{s}(t, \phi) \leqslant \sum_{i=1}^{m} w_{i s}(t, V)
$$

for all interconnection matrices $E_{t}$ and $s \in I_{m_{0}}$. Therefore,

$$
D_{(2)} V(t, \phi) \leqslant \bar{w}(t, V(t, \phi))
$$

By inequalities (13), (14), hypothesis iv) and Theorem 1, we know that the zero solution of system (2) is connectively and uniformly asymptotically stable, and this completes the proof.

Theorem 3: If the following conditions are satisfied:

i) eash isolated subsystem (4) possesses property $A_{1}$;

ii) there exist constants $a_{i j} \in R$ such that

$$
D_{(2)} V_{i s}\left(t, \phi^{i}\right) \leqslant D_{(4)} V_{i s}\left(t, \phi^{i}\right)
$$

$$
+\left(W_{i 3}\left(\left\|\phi_{j}\right\|\right)\right)^{1 / 2} \sum_{j=1}^{m}\left\|\bar{e}_{t}^{i j}\right\| a_{i j}\left(W_{j 3}\left(\left\|\phi^{j}\right\|\right)\right)^{1 / 2}
$$

for all interconnection matrices $E_{t}$;

iii) the $m \times m$ text matrix $S=\left(s_{i j}\right)$ defined by

$$
s_{i j}= \begin{cases}\sigma_{i}+\left\|\bar{e}_{t}^{i i}\right\| a_{i i} & i=j \\ 1 / 2\left(\left\|e_{t}^{i j}\right\| a_{i j}+\left\|e_{t}^{j i}\right\| a_{j i}\right) & i \neq j\end{cases}
$$

is negative definite;

iv) the zero solution of comparison system (12) is uniformly asymptotically stable.

Then the zero solution of large scale system (1) with decomposition (2) and (3) is connectively and uniformly asymptotically stable.

The proof of Theorem 3 is similiar to that of Theorem 2; here we omit

\section{EXAMPLE}

Consider a large scale system described by the delay equation

$$
\dot{x}^{i}(t)=-\frac{1}{e} x^{i}(t-\tau)+\sum_{j=1}^{m} e_{1}^{i j} a_{i j}(t) x^{j}(t-\tau), \quad \tau>0
$$


The isolated subsystem of (15) has the form

$$
\dot{x}^{i}(t)=-\frac{1}{e} x^{i}(t-\tau)
$$

and subsystem (16) can be written in the integral form as

$$
x^{i}\left(t ; t_{0}, \phi\right)=Y_{i}(0, t) \phi^{i}(0)-\frac{1}{e} \int_{-\tau}^{0} Y_{i}(s+\tau, t) \phi^{i}(s) d s
$$

where $Y_{i}(s, t)$ is defined as

$$
Y_{i}(s, t)= \begin{cases}\exp [-(t-s)] & \text { when } s<t \\ 1 & \text { when } \dot{s}=t \\ 0 & \text { when } t<s \leqslant t+1 .\end{cases}
$$

Now define a functional

$$
V_{i}\left(t, \phi^{i}\right)=\lim _{s \geqslant 0}\left[\left\|x_{t+s}^{i}\left(t_{0}, \phi\right)\right\|\right] \exp \left(\lambda_{i} s\right) ;
$$

one can show, following Lakshmikantham and Leela [5], that

$$
\left\|V_{i}\left(t, \phi_{1}^{i}\right)-V_{i}\left(t, \phi_{2}^{i}\right)\right\| \leqslant L_{i}\left\|\phi_{1}^{i}-\phi_{2}^{i}\right\| .
$$

The continuity of $V_{i}\left(t, \phi^{i}\right)$ can easily be proved, and

$$
\begin{aligned}
& D_{(16)} V_{i}\left(t, \phi^{i}\right)=\lim \sup _{h \rightarrow 0^{+}} 1 / h\left\{\operatorname { s u p } _ { s \geqslant 0 } \left[\left\|x_{t+h+s}^{i}\left(t+h, x_{t+h}^{i}\left(t_{0}, \phi\right)\right)\right\|\right.\right. \\
& \left.\left.-\left\|x_{t+s}^{i}\left(t_{0}, \phi\right)\right\|\right] \exp \left(\lambda_{i} s\right)\right\} \\
& =\lim _{h \rightarrow 0^{+}} \sup 1 / h\left\{\operatorname { s u p } _ { s \geqslant 0 } \left[\left\|x_{t+s}^{i}\left(t_{0}, \phi\right)\right\| \exp \left(\lambda_{i}(s-h)\right)\right.\right. \\
& \left.\left.-\left\|x_{t+h}^{i}\left(t_{0}, \phi\right)\right\| \exp \left(\lambda_{i} s\right)\right]\right\} \\
& \leqslant V_{i}\left(t, \phi^{i}\right) \lim _{h \rightarrow 0^{+}} \sup 1 / h\left[\exp \left(-\lambda_{i} h\right)-1\right] \\
& \leqslant-\lambda_{i} W_{i 3}\left(\left\|\phi^{i}\right\|\right)
\end{aligned}
$$

which shows that the isolated subsystem (16) possesses property $A$ with $w_{i}$ $=0$. And

$$
\begin{aligned}
\left\|g_{i}(t, \phi)\right\| & \leqslant \sum_{j=1}^{m}\left\|\bar{e}_{t}^{i j}\right\|\left\|a_{i j}(t)\right\|\left\|x^{j}(t-\tau)\right\| \\
& \leqslant \sum_{j=1}^{m}\left\|\bar{e}_{t}^{i j}\right\|\left\|\bar{a}_{i j}\right\|\left\|V_{j}\left(t, \phi^{j}\right)\right\| \\
& \leqslant\left(W_{i 3}\left(\left\|\phi^{j}\right\|\right)\right)^{1 / 2} \sum_{j=1}^{m}\left\|\bar{e}_{t}^{i j}\right\|\left\|\bar{a}_{i j}\right\|\left(W_{j 3}\left(\left\|\phi^{j}\right\|\right)\right)^{1 / 2} .
\end{aligned}
$$

The $m \times m$ text matrix $S=\left(s_{i j}\right)$ is defined by

$$
s_{i j}= \begin{cases}\lambda_{i}-L_{i}\left\|\bar{e}_{t}^{i i}\right\|\left\|\bar{a}_{i i}\right\| & i=j \\ -1 / 2\left(L_{i}\left\|\bar{e}_{t}^{i j}\right\|\left\|\bar{a}_{i j}\right\|+L_{j}\left\|\bar{e}_{t}^{-j i}\right\|\left\|\bar{a}_{j i}\right\|\right) & i \neq j .\end{cases}
$$

Therefore, if $S$ has positive successive principal minors, then by Theorem 2 the zero solution of system (15) is connectively and asymptotically stable.

\section{ACKNOWLEDGMENT}

The author would like to thank Prof. Liao Xiaoxin and Prof. Deng Zhongqi for their helpful comments. Thanks are also due to the referee.

\section{REFERENCES}

[1] F. N. Bailey "The application of Liapunov's second method to interconnected systems," SIAM J. Contr., vol. 3, pp. 433-462, 1966.

21 Lj. T. Grujic and D. D. Siljak. "Asymptotic stability and instability of large scale systems," IEEE Trans. Automat. Contr., vol. AC-18, pp. 636-645, 1973.
[3] J. K. Hale, Theory of Functional Differential Equations. New York: Springer-Verlag, 1977

[4] G. S. Ladde, "Systems of functional differential inequalities and functional differential systems," Pacific J. Math., vol. 66, pp. 161-171, 1976

[5] V. Lashmikantham and S. Leela, Differential and Integral Inequalities. New York: Academic, 1969.

[6] A. N. Michel, "Stability analysis of interconnected systems," SIAM J. Contr., vol. 12 , pp. 554-579, 1974

[7] A. N. Michel and R. K. Miller, Qualitative Analysis of Large Scale Dynamic Systems. New York: Academic, 1977.

[8] D. D. Siljak, "Stability of large scale systems under structural perturbations," IEEE Trans. Syst., Man, Cybern., vol. SMC-2, pp. 657-663, 1972

[9] D. D. Siljak, Large Scale Dynamic Systems: Stability and Structure. New York: North-Holland, 1978.

[10] Cai Weixuan, "Stability of composite systems with time delay," J. Xiamen Univ., vol. 21, pp. 249-260, 1982

\section{A New Controller Design for Manipulators Using the Theory of Variable Structure Systems}

\author{
KAI S. YEUNG AND YON P. CHEN
}

Abstract-A new controller design of manipulators using the theory of VSS is presented to deal with the set-point regulation problem. The major obstacle of VSS vector control with strong dynamic coupling is overcome for a class of systems with positive definite symmetric inertia matrices. Parameter variations can easily be considered in the design methodology, which is easily extendable to a higher number of links. The problem of chattering is solved by the introduction of sliding sectors.

\section{INTRODUCTION}

In the conventional controller design for robotic manipulators, the control algorithm is based on nonlinear compensations of the plant. This approach requires a detailed model of the manipulator and an exact load forecast [1]. In addition, such nonlinear compensations are complex and costly to implement. In order to avoid this difficulty, control algorithms using the theory of variable structure systems (VSS) have been developed [2]-[4]. The variable structure system is designed in such a way that all trajectories in the state space are directed toward some switching planes [5], [6]. Once the system state reaches the switching planes, it slides along them and the system response depends thereafter only on the gradients of the switching planes and remains insensitive to a class of disturbances and parameter variations [7].

Among developed control algorithms using the theory of VSS, there still exist some nontrivial difficulties in the design. A major difficulty is related to the cross-coupling in the inertia matrix. To get around this difficulty, Young [2] uses the hierarchical approach and Morgan et al. [3] employ dynamic coupling compensation. Both approaches are not entirely satisfactory in that the control torques are excessive. Recent work by Slotine et al. [4] is faced with similar difficulties. The need to invert the inertia matrix makes the extension of the design to a higher number of links difficult. Another unfavorable feature of the existing VSS design is the large amount of chattering which is associated with the excessive control torques.

In this note, we propose a new control algorithm which takes advantage of an important property of the inertia matrix, namely its symmetric positive-definiteness [8]. Consideration of this property allows a development of the control law without having to take the inverse of the inertia

Manuscript received May 7, 1986; revised May 27, 1987. Paper recommended by Past Associate Editor, W. J. Book. This work was supported by the Automation and Robotics Research Institute, University of Texas at Arlington, Arlington, TX.

The authors are with the Department of Electrical Engineering, The University of Texas at Arlington, Arlington, TX 76019.

Texas at Arington, Arlington,
IEEE Log Number 8716721 . 